\title{
Regional Ionospheric VTEC Estimation Applied by Spherical Cap Harmonic Analysis and Kalman Filter
}

\author{
M. Ohashi, K. Nishimoto, Y. Kubo and S. Sugimoto \\ Department of Electrical and Electronic Engineering, Ritsumeikan University \\ Noji-Higashi, Kusatsu City, Shiga 525-8577 Japan \\ Tel: +81-(0)77-561-5972, FAX: +81-(0)77-561-2663 \\ E-mail: ykubo@se.ritsumei.ac.jp
}

\begin{abstract}
In this paper, the regional ionospheric VTEC models for GNSS (Global Navigation Satellite System) positioning in the local area like as Japan are investigated. The ionosphere is modeled based on VTEC (Vertical Total Electron Content) estimation methods. In order to model regional VTEC distribution from regional data, GEONET (Gnss Earth Observation NETwork) data are used and obtained from the GSI (Geospatial Information Authority of Japan). In our previous research, Least Squares method has been applied. In this paper, we propose the method by applying Kalman Filter. In the experiments, the ionospheric VTEC is modeled by the previous and proposed methods.
\end{abstract}

\section{Introduction}

The ionospheric delay is dominant error cause of GNSS (Global Navigation Satellite System) positioning. Thus, high positioning accuracy requires accurate correction of ionospheric delay. The ionosphere is modeled based on the ionospheric VTEC (Vertical Total Electron Content) estimation methods. In the methods, the VTEC distribution is expressed by using SCHA (Spherical Cap Harmonic Analysis) in [1]. In our previous research, the SCHA coefficients are estimated by Least Squares method from the data at all epochs within 2 hours because the estimate cannot be calculated at an epoch. Thus, the time interval of the previous model is 2 hours. In this paper, the estimation method by applying Kalman Filter is proposed so that the time resolution of the ionospheric model is refined.

In section 2, the fundamental equations for GNSS positioning are shown. These equations are transformed to model the VTEC. In section 3, the single-layer model assuming ionosphere as single spherical shell is shown. The regional VTEC of the single-layer model can be modeled with SCHA. In section 4 , the 2 estimation methods of the ionospheric delay are described. One is the previous method by applying Least Squares method. Another one is the proposed method by applying Kalman Filter. In section 5, the VTEC is modeled from the GEONET (Gnss Earth Observation NETwork) data of the GSI (Geospatial Information Author- ity of Japan). Furthermore, the proposed VTEC model is compared with previous models.

\section{Fundamental Equations}

\subsection{GNSS Regression Model}

We consider the following fundamental measurements of the pseudoranges $\rho_{C A, k}^{p}, \rho_{P Y, k}^{p}$ based on $\mathrm{C} / \mathrm{A}, \mathrm{P}(\mathrm{Y})$ codes, and L1 and L2 band carrier phases $\Phi_{L 1, k}^{p}, \Phi_{L 2, k}^{p}$, respectively, for the known position $k$ as follows,

$$
\begin{aligned}
\rho_{C A, k}^{p}= & r_{k}^{p}+c\left(\delta t_{k}-\delta t^{p}\right)+\delta I_{k}^{p}+\delta T_{k}^{p} \\
& +\delta b_{C A, k}+\delta b_{C A}^{p}+e_{C A, k}^{p} \\
\rho_{P Y, k}^{p}= & r_{k}^{p}+c\left(\delta t_{k}-\delta t^{p}\right)+\frac{f_{1}{ }^{2}}{f_{2}{ }^{2}} \delta I_{k}^{p}+\delta T_{k}^{p} \\
& +\delta b_{P Y, k}+\delta b_{P Y}^{p}+e_{P Y, k}^{p} \\
\Phi_{L 1, k}^{p}= & r_{k}^{p}+c\left(\delta t_{k}-\delta t^{p}\right)-\delta I_{k}^{p}+\delta T_{k}^{p}+\lambda_{1} N_{L 1, k}^{p} \\
& +\delta b_{L 1, k}+\delta b_{L 1}^{p}+\varepsilon_{L 1, k}^{p} \\
\Phi_{L 2, k}^{p}= & r_{k}^{p}+c\left(\delta t_{k}-\delta t^{p}\right)-\frac{f_{1}{ }^{2}}{f_{2}{ }^{2}} \delta I_{k}^{p}+\delta T_{k}^{p}+\lambda_{2} N_{L 2, k}^{p} \\
& +\delta b_{L 2, k}+\delta b_{L 2}^{p}+\varepsilon_{L 2, k}^{p}
\end{aligned}
$$

where $\mathrm{c}\left(\approx 2.99792458 \times 10^{8}[\mathrm{~m} / \mathrm{s}]\right)$ denotes the speed of light, $f_{i}$ and $\lambda_{i}$ are the central frequency and the wave length of the $L_{i}$ carrier wave, $f_{1}=1575.42[\mathrm{MHz}], f_{2}=$ $1227.60[\mathrm{MHz}]$. In eqs. (1)-(4), the so-called receiver's hardware biases, $\left\{\delta b_{C A, k}, \delta b_{P Y, k}, \delta b_{L 1, k}, \delta b_{L 2, k}\right\}$, and the satellite's hardware biases, $\left\{\delta b_{C A}^{s^{p}}, \delta b_{P Y}^{s^{p}}, \delta b_{L 1}^{s^{p}}, \delta b_{L 2}^{s^{p}}\right\}$, are contained in the usual observed positioning data. Also, $r_{k}^{p}$ is the geometric distance between the receiver $k$ and the satellite $p\left(p=1, \cdots, n_{s}\right)$. Namely,

$$
r_{k}^{p}=\sqrt{\left(x_{k}-x^{p}\right)^{2}+\left(y_{k}-y^{p}\right)^{2}+\left(z_{k}-z^{p}\right)^{2}}
$$

where $k \equiv\left[x_{k}, y_{k}, z_{k}\right]^{\mathrm{T}}$ and $s^{p} \equiv\left[x^{p}, y^{p}, z^{p}\right]^{\mathrm{T}}$ are the known receiver position and the $p$-th satellite position, respectively. $n_{s}$ is the number of observable satellites. Further in (1)-(4), $\delta I_{k}^{p}, \delta T_{k}^{p}$ reflect the delay or the advance associated with the transmission of the $L_{1}$ signal through the ionosphere and the troposphere, respectively. $\delta t_{k}, \delta t^{p}$ are the clock errors of the receiver $k$ and the satellite $p . N_{L_{i}, k}^{p}$ denotes the phase ambiguity between the satellite $p$ and the receiver $k$ in $L_{i}$ band, 
and $e, \varepsilon$ denote measurement errors.

We can then express eqs. (1)-(4) as the following vector regression equation for $n_{s}$ satellites.

$$
\boldsymbol{y}_{k}=H_{k} \boldsymbol{\theta}_{k}+\boldsymbol{v}_{k}
$$

where

$$
\begin{aligned}
& \boldsymbol{y}_{k} \equiv\left[\boldsymbol{\rho}_{C A, k}^{\mathrm{T}}, \boldsymbol{\rho}_{P Y, k}^{\mathrm{T}}, \boldsymbol{\Phi}_{L 1, k}^{\mathrm{T}}, \boldsymbol{\Phi}_{L 2, k}^{\mathrm{T}}\right]^{\mathrm{T}}
\end{aligned}
$$

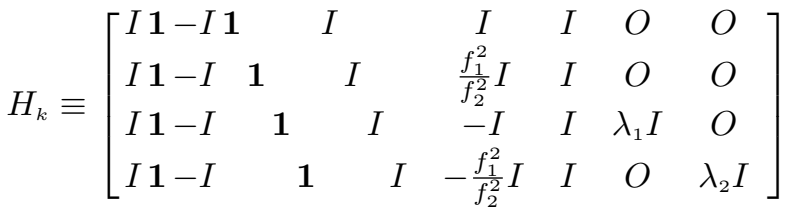

$$
\begin{aligned}
& \boldsymbol{\theta}_{k} \equiv\left[\boldsymbol{r}_{k}^{\mathrm{T}}, c \delta t_{k}, \boldsymbol{c} \boldsymbol{\delta} \boldsymbol{t}^{s \mathrm{~T}}, \boldsymbol{\delta} \boldsymbol{b}_{k}{ }^{\mathrm{T}}, \boldsymbol{\delta} \boldsymbol{b}^{s \mathrm{~T}},\right. \\
& \left.\boldsymbol{\delta} \boldsymbol{I}_{k}^{\mathrm{T}}, \boldsymbol{\delta} \boldsymbol{T}_{k}^{\mathrm{T}}, \boldsymbol{N}_{L 1, k}^{\mathrm{T}}, \boldsymbol{N}_{L 2, k}^{\mathrm{T}}\right]^{\mathrm{T}} \\
& \boldsymbol{\delta} \boldsymbol{b}_{k} \equiv\left[\delta b_{C A, k}, \delta b_{P Y, k}, \delta b_{L 1, k}, \delta b_{L 2, k}\right]^{\mathrm{T}}
\end{aligned}
$$

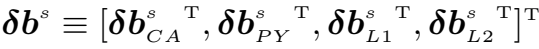

$$
\begin{aligned}
& \boldsymbol{v}_{k} \equiv\left[\boldsymbol{e}_{C A, k}^{\mathrm{T}}, \boldsymbol{e}_{P Y, k}^{\mathrm{T}}, \boldsymbol{\varepsilon}_{L 1, k}^{\mathrm{T}}, \boldsymbol{\varepsilon}_{L 2, k}^{\mathrm{T}}\right]^{\mathrm{T}}
\end{aligned}
$$

and $\mathbf{1} \equiv[1, \cdots, 1], n_{s} \times 1$ vector, $I$ denote the $n_{s} \times n_{s}$ unit matrix.

\subsection{Preprocessing on measurement data}

In this paper, the ionospheric term $\boldsymbol{\delta} \boldsymbol{I}_{k}$ in (6) is focused on, and the ionospheric models are derived in the following sections. For this purpose, the unknown parameters in (6) can be greatly reduced by taking socalled the geometry-free combination [2]. Then, the geometry-free operation on (6) can be expressed as follows,

$$
D \boldsymbol{y}_{k}=D H_{k} \boldsymbol{\theta}_{k}+D \boldsymbol{v}_{k}
$$

where

$$
D \equiv\left[\begin{array}{cccc}
-I & I & O & O \\
O & O & -I & I
\end{array}\right]
$$

Furthermore, we have

$$
\begin{aligned}
& {\left[\begin{array}{l}
\boldsymbol{\rho}_{P Y, k}-\boldsymbol{\rho}_{C A, k} \\
\boldsymbol{\Phi}_{L 2, k}-\boldsymbol{\Phi}_{L 1, k}
\end{array}\right]} \\
& =\left[\begin{array}{ccc}
I & O & \left(\frac{f_{1}^{2}}{f_{2}^{2}}-1\right) I \\
O & I & -\left(\frac{f_{1}^{2}}{f_{2}^{2}}-1\right) I
\end{array}\right]\left[\begin{array}{c}
\boldsymbol{B}_{C A-P Y, k} \\
\boldsymbol{B}_{L 1-L 2, k} \\
\boldsymbol{\delta} I_{k}
\end{array}\right]+\left[\begin{array}{c}
\boldsymbol{v}_{P Y, k}-\boldsymbol{v}_{C A, k} \\
\boldsymbol{v}_{L 2, k}-\boldsymbol{v}_{L 1, k}
\end{array}\right]
\end{aligned}
$$

where

$$
\begin{aligned}
\boldsymbol{B}_{C A-P Y, k}= & \mathbf{1}\left(\boldsymbol{\delta} \boldsymbol{b}_{P Y, k}-\boldsymbol{\delta} \boldsymbol{b}_{C A, k}\right)+\boldsymbol{\delta} \boldsymbol{b}_{P Y}^{s}-\boldsymbol{\delta} \boldsymbol{b}_{C A}^{s}(10) \\
\boldsymbol{B}_{L 1-L 2, k}= & \mathbf{1}\left(\boldsymbol{\delta} \boldsymbol{b}_{L 2, k}-\boldsymbol{\delta} \boldsymbol{b}_{L 1, k}\right)+\boldsymbol{\delta} \boldsymbol{b}_{L 2}^{s}-\boldsymbol{\delta} \boldsymbol{b}_{L 1}^{s} \\
& +\lambda_{2} \boldsymbol{N}_{L 2, k}-\lambda_{1} \boldsymbol{N}_{L 1, k}
\end{aligned}
$$

By the transformations, geometric distance $\boldsymbol{r}_{k}$, tropospheric delay $\boldsymbol{\delta} \boldsymbol{T}_{k}$, the clock errors of the receiver and the satellite $\delta t_{k}, \boldsymbol{\delta} \boldsymbol{t}^{s}$ are eliminated.

\section{Ionospheric VTEC Model}

\subsection{Single-Layer Model}

In this section, we develop an estimation method of SCHA models of the local VTEC map in the sky over Japan. We here apply the single-layer model shown

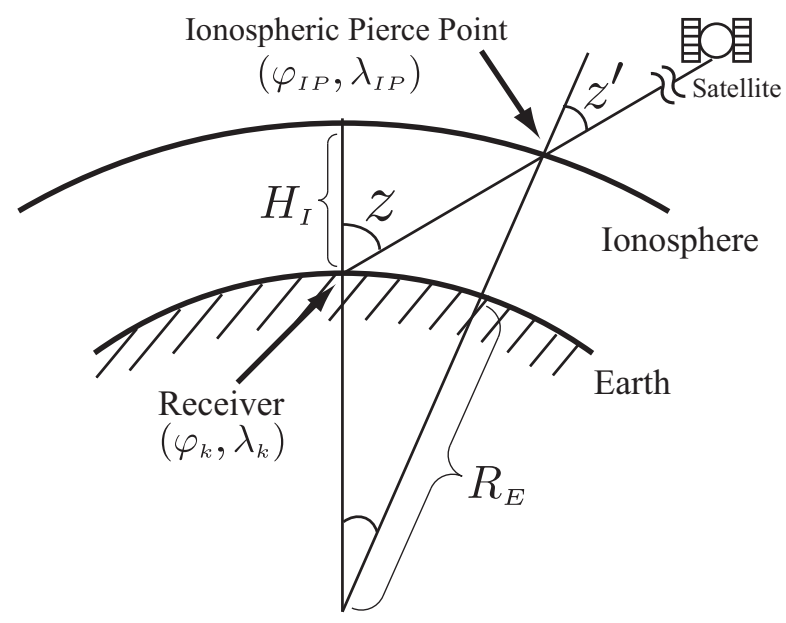

Fig. 1: Single-Layer model

in Fig. 1. In this model, the ionosphere is modeled by a thin shell. Because GPS basically provides measurements of the ionospheric delay(or advance) between the satellite and the receiver in meter due to the slant TEC instead of VTEC, it is important to formulate the relationship between the measured ionospheric delay and VTEC. Therefore, an elevation-dependent mapping function $M_{I}$ which transforms VTEC to the slant TEC at the IPP (Ionospheric Pierce Point) as follows,

$$
M_{I}=\frac{1}{\cos z^{\prime}}
$$

where $z^{\prime}$ is the satellite's zenith angle at the IPP. The relationship between $z$ and $z^{\prime}$ is expressed by

$$
z^{\prime}=\arcsin \left(\frac{R_{E}}{R_{E}+H_{I}} \sin z\right)
$$

where $R_{E}(\approx 6371[\mathrm{~km}])$ is the mean earth radius and $H_{I}$ is the height of single-layer. Then we have the relationship between $\delta I$ and VTEC as follows [4],

$$
\delta I=\frac{40.3 \times 10^{16}}{f^{2}} \times M_{I} \times \mathrm{VTEC} .
$$

\subsection{Spherical Cap Harmonics Analysis (SCHA) Model}

In order to model the distribution of the VTEC, several methods such as power series expansion, SCHA have been proposed. SCHA is the harmonic function around a part of sphere and the local VTEC such as in the sky over Japan is focused on in this paper. Therefore, also from results of previous works $[1,5,6]$, it can be considered the VTEC is well modeled by SCHA. 
Now, let $\phi_{I P}$ and $\lambda_{I P}$ be the latitude and longitude of the IPP, then the VTEC at the IPP, $E\left(\phi_{I P}, \lambda_{I P}\right)$, is modeled by SCHA as follows $[1,5]$,

$$
\begin{aligned}
\mathrm{VTEC}= & E\left(\phi_{I P}, \lambda_{I P}\right) \\
= & \sum_{l=0}^{n_{\max }} \sum_{m=0}^{l}\left\{\left(a_{l m} \cos m \lambda_{I P}+b_{l m} \sin m \lambda_{I P}\right)\right. \\
& \left.\times P_{n_{l}(m)}^{m}\left(\sin \phi_{I P}\right)\right\} .
\end{aligned}
$$

where $a_{l m}$ and $b_{l m}$ are coefficients of SCHA, the parameters $l$ and $m$ are generally referred to as the degree and order, $n_{\max }$ denotes the maximum degree of SCHA, $P_{n_{l}(m)}^{m}$ is the normalized associated extended Legendre function in SCHA. The real degrees $n_{l}$ are the functions of the orders $m$ and the detailed solution can be found in [7]. Combining eqs. (13) and (14), we have the relationship

$$
\begin{aligned}
\delta I_{k}^{p}= & \frac{40.3 \times 10^{16}}{f_{1}^{2}} \times\left[\frac{1}{\cos \left(z_{k}^{p}\right)^{\prime}} \times \sum_{l=0}^{n_{\max }} \sum_{m=0}^{l}\right. \\
& \left.\left\{a_{l m} \cos m \lambda_{I P}+b_{l m} \sin m \lambda_{I P}\right\} P_{n_{l(m)}}^{m}\left(\sin \phi_{I P}\right)\right] .
\end{aligned}
$$

By using eq. (16), we can formulate the ionospheric delay $\delta I_{k}$ for satellites $p=1, \cdots, n_{s}$ and receiver $k$ as follows,

$$
\boldsymbol{\delta} \boldsymbol{I}_{k}=\left[\begin{array}{c}
\delta I_{k}^{1} \\
\vdots \\
\delta I_{k}^{n_{s}}
\end{array}\right]=\left[\begin{array}{ll}
A_{a, k} & A_{b, k}
\end{array}\right]\left[\begin{array}{c}
\boldsymbol{\theta}_{a} \\
\boldsymbol{\theta}_{b}
\end{array}\right]
$$

where

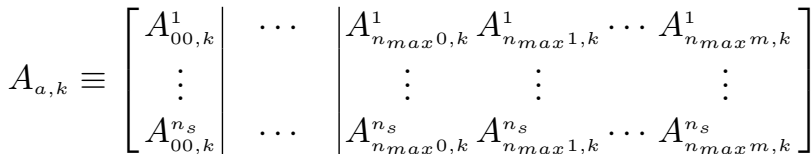

$$
\begin{aligned}
& A_{b, k} \equiv\left[\begin{array}{c|c|cccc}
B_{00, k}^{1} & \cdots & B_{n_{\max 0, k}}^{1} & B_{n_{\max 1, k}}^{1} & \cdots & B_{n_{\max m, k}}^{1} \\
\vdots & & \vdots & \vdots & \vdots \\
B_{00, k}^{n_{s}} & \cdots & B_{n_{\max } n_{s}}^{n_{s}} & B_{n_{\max } n_{s}}^{n_{s}} & \cdots & B_{n_{\max } n_{s}}^{n_{s}}
\end{array}\right] \\
& A_{n m, k}^{p}=\frac{40.3 \times 10^{16}}{f_{1}^{2}} \times \frac{1}{\cos \left(z_{k}^{p}\right)^{\prime}} \\
& \times\left(\cos m \lambda_{I P}\right) P_{n_{l(m)}}^{m}\left(\sin \phi_{I P}\right) \\
& B_{n m, k}^{p}=\frac{40.3 \times 10^{16}}{f_{1}^{2}} \times \frac{1}{\cos \left(z_{k}^{p}\right)^{\prime}} \\
& \times\left(\sin m \lambda_{I P}\right) P_{n_{l(m)}}^{m}\left(\sin \phi_{I P}\right) \\
& \boldsymbol{\theta}_{a} \equiv\left[\begin{array}{l|l|lll}
a_{00}|\cdots| a_{n_{\max } 0} & a_{n_{\max 1}} & \cdots & a_{n_{\max } m}
\end{array}\right]^{\mathrm{T}} \\
& \boldsymbol{\theta}_{b} \equiv\left[\begin{array}{l|l|llll}
b_{00} & \cdots & b_{n_{\max } 0} & b_{n_{\max 1}} & \cdots & b_{n_{\max m}}
\end{array}\right]^{\mathrm{T}} \text {. }
\end{aligned}
$$

Therefore, in order to model the VTEC by SCHA, the coefficients $\theta_{a}$ and $\theta_{b}$ should be estimated from the measurement of eq. (9).

\subsection{Measurement Equation with Single-Layer and SCHA Model}

By substituting $\boldsymbol{\delta} I_{k}$ of eq. (17) into eq. (9), the measurement equation is expressed as follows,

$$
\boldsymbol{y}_{d, k}=H_{d, k} \boldsymbol{\theta}_{d, k}+\boldsymbol{v}_{d, k}
$$

where the matrix $H_{d, k}$ and the vector $\boldsymbol{\theta}_{d, k}$ are redefined as follows,

$$
\begin{aligned}
& H_{d, k} \equiv\left[\begin{array}{l|ll}
A_{k} & I_{n_{s}} & \\
& & I_{n_{s}}
\end{array}\right], \\
& A_{k} \equiv\left[\begin{array}{c}
\left(\frac{f_{1}^{2}}{f_{2}^{2}}-1\right) I_{n_{s}} \\
-\left(\frac{f_{1}^{2}}{f_{2}^{2}}-1\right) I_{n_{s}}
\end{array}\right]\left[\begin{array}{ll}
A_{a, k} & A_{b, k}
\end{array}\right] \text {, } \\
& \boldsymbol{\theta}_{d, k} \equiv\left[\begin{array}{c}
\boldsymbol{\theta}_{c} \\
\boldsymbol{B}_{P Y-C A, k} \\
\boldsymbol{B}_{L 2-L 1, k}
\end{array}\right], \boldsymbol{\theta}_{c} \equiv\left[\begin{array}{c}
\boldsymbol{\theta}_{a} \\
\boldsymbol{\theta}_{b}
\end{array}\right] \text {. }
\end{aligned}
$$

In actual, the number of visible satellites depends on the position of the receiver and time. In this paper, it is assumed that the number of visible satellites at each receiver is the same. From eq. (18), when we collect all of the receiver data from $n_{s}$ satellites and $M$ GEONET stations $1, \cdots, M$, we have

$$
\boldsymbol{Y}_{M}=H_{M} \boldsymbol{\theta}_{M}+\boldsymbol{v}_{M}
$$

where

$$
\begin{gathered}
\boldsymbol{Y}_{M} \equiv\left[\begin{array}{c}
\boldsymbol{y}_{d, 1} \\
\vdots \\
\boldsymbol{y}_{d, M}
\end{array}\right], H_{M} \equiv\left[\begin{array}{ccc}
A_{1} & I_{2 n_{s}} & \\
\vdots & \ddots & \\
A_{M} & & I_{2 n_{s}}
\end{array}\right] \\
\boldsymbol{\theta}_{M} \equiv\left[\begin{array}{c}
\boldsymbol{\theta}_{c} \\
\boldsymbol{B}_{P Y-C A, 1} \\
\boldsymbol{B}_{L 2-L 1,1} \\
\vdots \\
\boldsymbol{B}_{P Y-C A, M} \\
\boldsymbol{B}_{L 2-L 1, M}
\end{array}\right], v_{M} \equiv\left[\begin{array}{c}
v_{d, 1} \\
\vdots \\
v_{d, M}
\end{array}\right]
\end{gathered}
$$

and $I_{2 n_{s}}$ denote the $2 n_{s} \times 2 n_{s}$ unit matrix.

\section{Estimation Methods}

\subsection{Estimation Method Applying Least Squares Method}

Because the measurement of eq. (19) can be obtained at successive observation epochs, hereafter, the time " $t$ " is added to eq. (19) as subscripts. In eq. (19), the number of unknown parameters is grater than the number of measurements at an epoch. In order to solve the equation, the unknown parameters are supposed to be constant within a given time, Then, by applying the Least Squares method, we have the smoothing estimate $\hat{\theta}_{M, t}$ at time $t$ as follows,

$\hat{\boldsymbol{\theta}}_{M, t}=\left(\sum_{j=t-d}^{t+d} H_{M, j}^{\mathrm{T}} R_{M, j}^{-1} H_{M, j}\right)^{-1}\left(\sum_{j=t-d}^{t+d} H_{M, j}^{\mathrm{T}} R_{M, j}^{-1} Y_{M, j}\right)$ 
where

$$
R_{M, j}=\operatorname{Cov}\left[\boldsymbol{v}_{M, j}\right] .
$$

The interval of GEONET data used in this research is 30 seconds. In this research, time $d$ is set as 1 hour and the estimates are obtained with interval of 2 hours.

\subsection{Estimation Method Applying Kalman Fil- ter}

By the method of section 4.1, VTEC can be modeled every 2 hours. This time resolution is as same as GIM provided by IGS and CODE. The VTEC value at an arbitrary time can be computed by linear interpolation (described below). A linear interpolation is fast and simple computation, however, it is considered that the interpolation error is large in the condition of using data every 2 hours. In this research, the estimation method is proposed to improve this low time resolution by applying Kalman Filter.

In order to apply Kalman Filter, the state space model is discussed. The bias error terms are assumed to be constant models. Additionally the SCHA coefficients are supposed to conform random walk process as follows,

$$
\boldsymbol{\theta}_{c, t+1}=\boldsymbol{\theta}_{c, t}+\boldsymbol{w}_{c, t},
$$

and $\boldsymbol{w}_{c, t}$ is the process noise which is supposed to be Gaussian white noise. From the above, state space model is expressed as follows,

$$
\begin{aligned}
\boldsymbol{\theta}_{M, t+1} & =F_{M, t} \boldsymbol{\theta}_{M, t}+\boldsymbol{w}_{M, t} \\
\boldsymbol{Y}_{M, t} & =H_{M, t} \boldsymbol{\theta}_{M, t}+\boldsymbol{v}_{M, t}
\end{aligned}
$$

where

$\boldsymbol{w}_{M, t}=\left[\begin{array}{c}\boldsymbol{w}_{c, t} \\ \mathbf{0}_{2 n_{s}} \\ \vdots \\ \mathbf{0}_{2 n_{s}}\end{array}\right], \quad F_{M, t}=\left[\begin{array}{cccc}I & & & \\ & I_{2 n_{s}} & & \\ & & \ddots & \\ & & & I_{2 n_{s}}\end{array}\right]$.

In eqs. (23) and (24), $F_{M}$ is the state transition matrix and eq. (24) is equivalent to eq. (19). Applying Kalman Filter to eqs. (23)-(24), the estimate can be obtained with the following process.

$$
\begin{aligned}
\text { (Initialization) } & \\
\hat{\boldsymbol{\theta}}_{M, 0 /-1} & =\overline{\boldsymbol{\theta}}_{M, 0} \\
P_{0 /-1} & =\Sigma_{0} \\
\text { (Updating) } & \\
K_{t}= & P_{t / t-1} H_{t}^{\mathrm{T}}\left[H_{M, t} P_{t / t-1} H_{M, t}^{\mathrm{T}}+R_{M, t}\right]^{-1} \\
\hat{\boldsymbol{\theta}}_{M, t / t} & =\hat{\boldsymbol{\theta}}_{M, t / t-1}+K_{t}\left[\boldsymbol{Y}_{M, t}-H_{M, t} \hat{\boldsymbol{\theta}}_{M, t / t-1}\right] \\
P_{t / t} & =P_{t / t-1}-K_{t} H_{M, t} P_{t / t-1} \\
(\text { Prediction }) & \\
\hat{\boldsymbol{\theta}}_{M, t+1 / t} & =F_{M, t} \hat{\boldsymbol{\theta}}_{M, t / t} \\
P_{t+1 / t} & =F_{M, t} P_{t / t} F_{M, t}^{\mathrm{T}}+Q_{M, t}
\end{aligned}
$$

In eqs. (25)-(31), $P$ is the estimation error covariance and $K$ is Kalman gain. $\overline{\boldsymbol{\theta}}_{M, 0}$ and $\Sigma_{0}$ are the initial values of $\hat{\boldsymbol{\theta}}$ and $P$. The SCHA coefficients $\hat{\boldsymbol{\theta}}_{c, t / t}$ can be estimated by eq. (28). The estimated SCHA coefficients are obtained with interval of 30 seconds as compared to the method described in section 4.1. Finally, the VTEC can be calculated with $\hat{\boldsymbol{\theta}}_{c, t / t}$.

\section{Experimental Results}

\subsection{The estimate of SCHA coefficients}

Based on estimation methods in section 4.1 and 4.2, the SCHA coefficients were estimated to model the VTEC on 2011/01/03. The observation data were obtained from 34 GEONET stations which are located all over Japan shown in Fig. 2. The height of ionospheric layer was set as $350[\mathrm{~km}]$. The maximum order of SCHA was set as 2 .

The SCHA coefficients estimated by Least Squares method and Kalman Filter are summarized in Tables 1 and 2. The SCHA coefficients were estimated every only 2 hours by Least Squares method as shown in Table 1. On the other hand, the SCHA coefficients were estimated every 30 seconds when Kalman Filter was applied. The results every 2 hours are shown to compare with Least Squares method in Table 2.

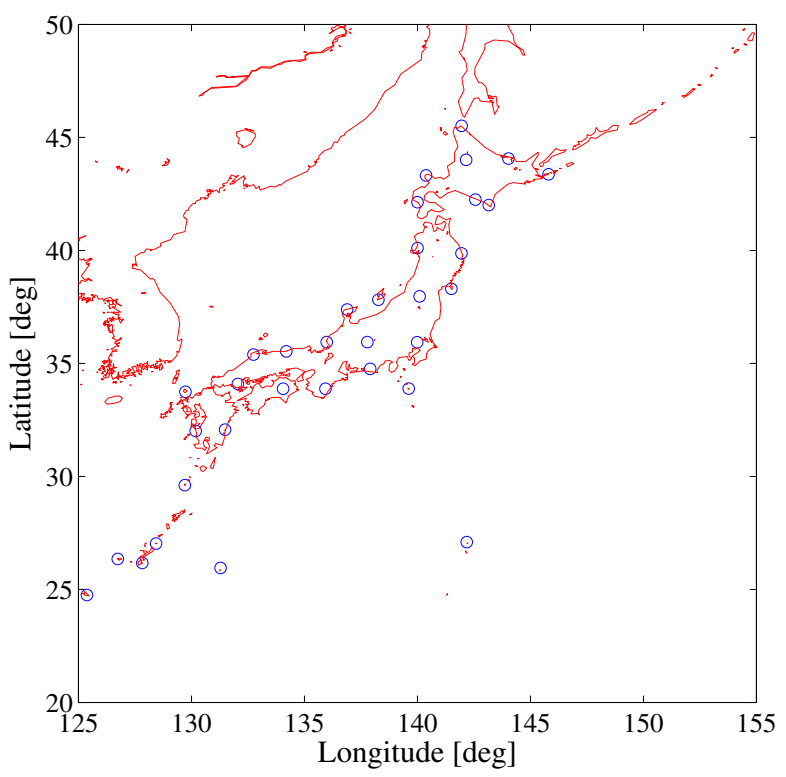

Fig. 2: GEONET stations used for estimation

\subsection{VTEC Maps}

Based on the SCHA coefficients shown in section 5.1, the VTEC maps the sky over Japan were produced on 2011/01/03. The VTEC maps by Least Squares method at 2 and 4 o'clock are shown in Figs. 3 and 4 . Similarly, the VTEC maps by Kalman Filter are shown in Figs. 5 and 6. The area where VTEC values are 
Table 1: SCHA coefficients estimated by Least Squares method

\begin{tabular}{|c|r|r|r|}
\hline Time & $00: 00: 00$ & $02: 00: 00$ & $04: 00: 00$ \\
\hline$a_{00}$ & 130.631 & 166.410 & 234.318 \\
\hline$a_{10}$ & 178.031 & 118.243 & 521.590 \\
\hline$a_{11}$ & -114.940 & -145.738 & -72.397 \\
\hline$a_{20}$ & 62.838 & 41.963 & 192.397 \\
\hline$a_{21}$ & -21.550 & -48.385 & 4.257 \\
\hline$a_{22}$ & -8.655 & -18.347 & 17.671 \\
\hline$b_{11}$ & -55.573 & -74.529 & -235.368 \\
\hline$b_{21}$ & -10.350 & -21.170 & -8.884 \\
\hline$b_{22}$ & -13.839 & -24.480 & -8.130 \\
\hline
\end{tabular}

Table 2: SCHA coefficients estimated by Kalman Filter

\begin{tabular}{|c|r|r|r|}
\hline Time & $00: 00: 00$ & $02: 00: 00$ & $04: 00: 00$ \\
\hline$a_{00}$ & 104.267 & 50.007 & 55.549 \\
\hline$a_{10}$ & 104.267 & -32.322 & -12.943 \\
\hline$a_{11}$ & 104.267 & -56.475 & -144.204 \\
\hline$a_{20}$ & 104.267 & -8.175 & -33.823 \\
\hline$a_{21}$ & 104.267 & 58.841 & 104.724 \\
\hline$a_{22}$ & 104.267 & -114.152 & -193.944 \\
\hline$b_{11}$ & 104.267 & 96.074 & 195.949 \\
\hline$b_{21}$ & 104.267 & -177.188 & -121.574 \\
\hline$b_{22}$ & 104.267 & -41.590 & 103.684 \\
\hline
\end{tabular}

negative, shown with white, can be observed on upper left of Fig. 6. This problem is due to the fact that VTEC is modeled by applying SCHA. In addition, it is considered that the observation condition is getting worse in consequence of the biased IPP distribution.

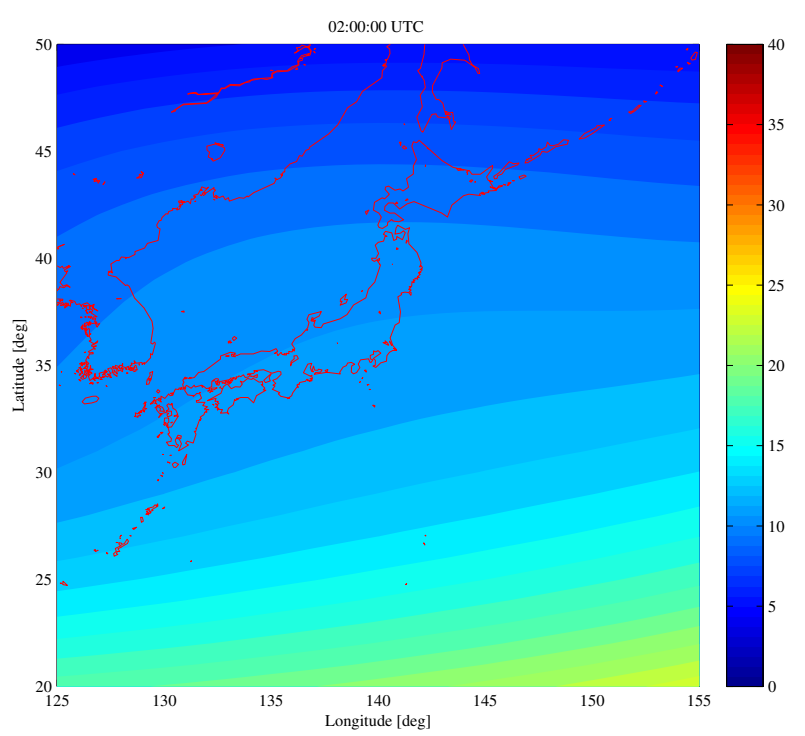

Fig. 3: VTEC maps by Least Squares method at 2 o'clock

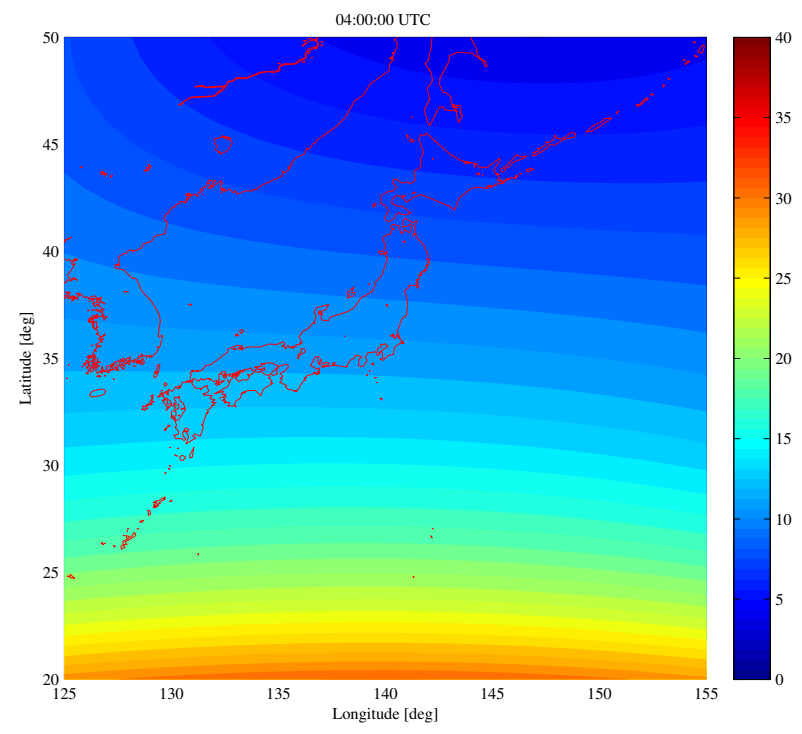

Fig. 4: VTEC maps by Least Squares method at 4 o'clock

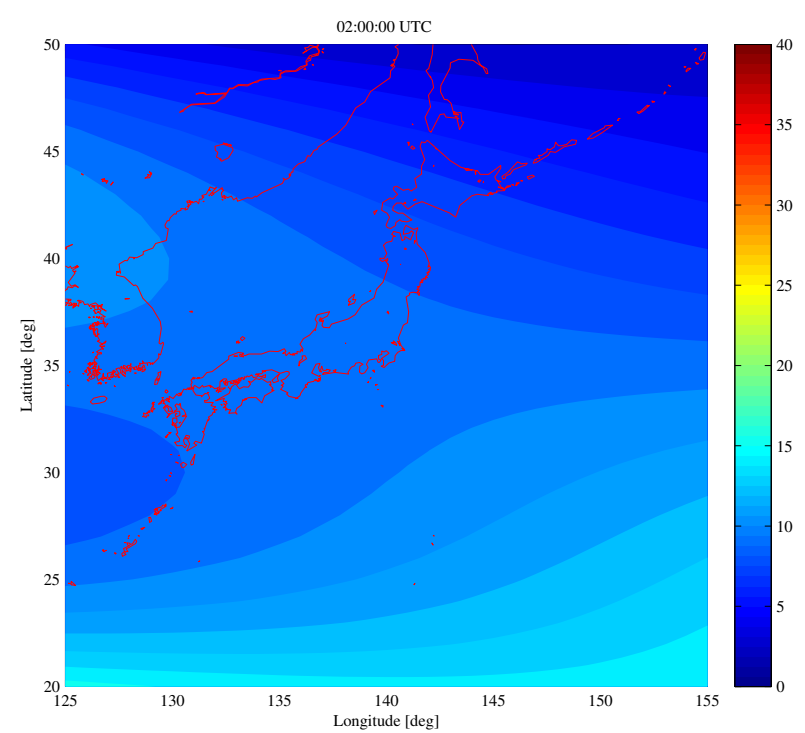

Fig. 5: VTEC maps by Kalman Filter at 2 o'clock

\section{Conclusions}

In this paper, the estimation method was proposed to model the regional ionospheric VTEC. The time interval of our conventional model with Least Squares method is 2 hours. In order to upgrade the time resolution, the estimation method with Kalman Filter was proposed. In the experiments, the model parameters were estimated by the proposed method from GEONET data. Then, the regional VTEC every 30 seconds could be modeled. In the future, we will apply the proposed model to positioning and evaluate that capability. 


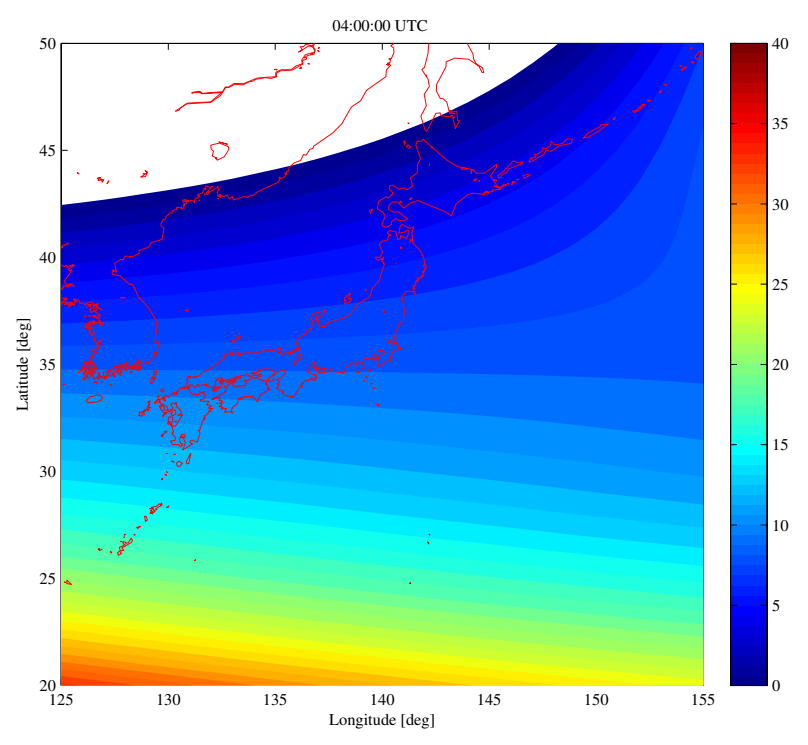

Fig. 6: VTEC maps by Kalman Filter at 4 o'clock

\section{References}

[1] S. Otsuki, M. Kamimura, M. Ohashi, Y. Kubo and S. Sugimoto: Local Models for Ionospheric VTEC Estimation Based on GR Models and Spherical Cap Harmonic Analysis, Journal of Aeronautics, Astronautics and Aviation, Vol. 43, No. 1, pp. 1-7 (2011).

[2] G. Xu: GPS - Theory, Algorithms and Applications, Springer-Verlag, Berlin (2003).

[3] Alfred Leick: GPS Satellite Surveying, 3rd Edition, Johon Wiley \& Sons (2003).

[4] B. W. Parkinson and J. J. Spilker Jr. (Eds.): Global Positioning System: Theory and Applications, Vol. I, II, AIAA (1997).

[5] M. Ohashi, T. Hattori, Y. Kubo and S. Sueo: Multi-Layer Ionospheric VTEC Estimation for GNSS Positioning Transactions of The Institute of Systems, Control and Information Engineers, Vol. 26, No. 1, pp. 16-24 (2013).

[6] S. Fujita, H. Yamamoto, T. Iura, Y. Kubo and S. Sugimoto: Modeling Ionosphere VTEC over Japan Based on GNSS Regression Models and GEONET, Int. J. of Innovative Computing, Information and Control, Vol. 6, No. 1, Jan., pp. 155-169 (2010).

[7] G. V. Haines: Spherical Cap Harmonic Analysis, Journal of Geophysical Reserach, Vol. 90, pp. 25932598 (1985).

[8] S. Schaer, W. Gurtner and J. Feltens: IONEX: The IONosphere Map EXchange format version 1, Proceedings of the IGS AC Workshop, Darmstadt, Germany (1998). 